To the Editors:

\title{
Spontaneous reports on anaphylaxis: the applicability of Brighton case definition
}

Ceylon Medical Journal 2011; 56: 129-130

Anaphylaxis is a clinical diagnosis with no gold standard confirmatory investigation. The need to have a uniformly accepted case definition for anaphylaxis led to the development of the Brighton definition [1] for anaphylaxis following immunizations (Box 1). It ascertains diagnostic certainty and classifies it into 3 levels. The level 1 classification is associated with the greatest diagnostic certainty. The objective of this study was to examine the applicability of the Brighton case definition to drug induced anaphylactic reactions.

The National Adverse Drug Reaction (ADR) monitoring centre for Sri Lanka which functions at the Department of Pharmacology, Faculty of Medicine, Colombo receives spontaneous ADR reports from health professionals. Spontaneous ADR reports on anaphylaxis received by this Centre since its inception in 1996 were retrieved from the database and used as the case reports to examine the applicability of Brighton case definition for drug induced anaphylaxis. The level of diagnostic certainty of these cases of drug induced anaphylaxis was determined using the Brighton case definition of anaphylaxis. Standard pharmacovigilance tools were used in further analysis. Naranjo Adverse Drug Reaction Probability Scale was used in assessing the causality [2]. Descriptive statistics was employed in presenting the results.

Over the period of 13 years, 58 cases have been reported as anaphylaxis. Of these, 39 (sensitivity $=67 \%$ ) fulfilled the Brighton case definition for anaphylaxis; 12 with level 1, 23 with level 2 and 4 with level 3 diagnostic certainty. The mean age of the patients was 36 years with male to female ratio of 1.3:1. Antimicrobials accounted for $62 \%$ followed by immunological products $(20 \%)$, antithrombotic medicines $(6.9 \%)$ and colloids (3.4\%). Thirdgeneration cephalosporins accounted for one-third of the cases. Causality was probable in $95 \%$ of reports. Case fatality rate was 22.4. Streptokinase (3 deaths), 3rd generation cephalosporins ( 3 deaths), anti snake venom (3 deaths) benzathine penicillin ( 2 deaths), oxytocin (1 death) and MMR vaccine (1 death) were the agents implicated in fatal drug induced anaphylaxis.

Cardiovascular, respiratory, dermatological and gastrointestinal symptoms were documented, respectively, in $86 \%, 55 \%, 22 \%$ and $21 \%$. The majority of cases $(93 \%)$ occurred following an injection. The mean duration between drug administration and onset of first symptom was 29 minutes (range $=2,120)$. The majority $(67 \%)$ of reports was from the doctors. Pharmacists accounted for $28 \%$ of the reports. Almost equal proportion of reports from doctors ( $72 \%$ ) and pharmacists (69\%) met the Brighton case definition for anaphylaxis following immunisation.

The Brighton case definition for anaphylaxis following immunisation is applicable with high sensitivity for spontaneous reports on drug induced anaphylaxis. However the number of reports assessed as level 2 diagnostic certainty is almost double the number of reports assessed as level 1 . This was mainly due to missing data in the reports because detailed records of clinical manifestations are required to apply the Brighton case definition for anaphylaxis. In the absence of detailed records of symptoms, chances are high that the anaphylaxis being classified as level 2 rather than level 1 . The routine 


\section{Box 1. Brighton case definition of anaphylaxis}

\section{For all levels of diagnostic certainty}

Anaphylaxis is a clinical syndrome characterized by

- Sudden onset AND

- Rapid progression of signs and symptoms AND

- involving multiple $(\geq 2)$ organ systems, as follows

\section{Level 1 of diagnostic certainty}

- $\geq 1$ major dermatological AND

- $\geq 1$ major cardiovascular AND/OR 1 major respiratory criterion

\section{Level 2 of diagnostic certainty}

- $\geq 1$ major cardiovascular AND $\geq 1$ major respiratory criterion OR

- $\geq 1$ major cardiovascular OR respiratory criterion AND

- $\geq 1$ minor criterion involving $\geq 1$ different system (other than cardiovascular or respiratory systems)

OR

- ( $\geq 1$ major dermatologic) AND ( $\geq 1$ minor cardiovascular AND/OR minor respiratory criterion)

\section{Level 3 of diagnostic certainty}

- $\geq 1$ minor cardiovascular OR respiratory criterion AND

- $\geq 1$ minor criterion from each of $\geq 2$ different systems/categories

ADR form if completed properly should capture all the information which is necessary to ascertain the diagnostic certainty. Since there will be no missing data in the reports, cases can be accurately classified as level 1 or level 2 diagnostic certainty. However given the workload and time constraints, health care personnel in Sri Lanka are unlikely to spontaneously report enough symptoms and signs to allow application of the Brighton case definition which was clearly evident in the ADR forms analysed.

A checklist, based on the terms used in the case definition, should be developed in order to remind health care personnel to note whether specific features of anaphylaxis were present. An anaphylaxis reporting form should be designed by incorporating this checklist into the routine ADR form, and used in spontaneous reporting of anaphylaxis. This would allow subsequent classification using the Brighton case definition and yield more reports with higher degree of diagnostic certainty.

Case fatality rate of $22.4 \%$ could be an overestimate as fatal cases would be reported more than the non fatal cases. However, it is higher than what is reported in the literature
(1\%) from many population based studies prompting further investigation and interventions $[3,4,5]$.

\section{References}

1. Ruggeberg JU, Gold M, Bayas JM, et al. The Brighton Collaboration Anaphylaxis Working Group.2 Anaphylaxis: case definition and guidelines for data collection, analysis, and presentation of immunization safety. Vaccine 2007; 25: 5675-84.

2. Naranjo CA, Busto U, Sellers EM, et al. A method for estimating the probability of adverse drug reactions. Clinical Pharmacology and Therapeutics 1981; 30:239-45.

3. Yocum MW, Butterfield JH, Klein JS, Volcheck GW, Schroeder DR, Silverstein MD. Epidemiology of anaphylaxis in Olmsted County: a population-based study. Journal of Allergy and Clinical Immunology 1999; 104: 452-6.

4. Brown AF, McKinnon D, Chu K. Emergency department anaphylaxis: A review of 142 patients in a single year. Journal of Allergy and Clinical Immunology 2001; 108: 861-6.

5. Bohlke K, Davis RL, DeStefano F, Marcy SM, Braun MM, Thompson RS. Epidemiology of anaphylaxis among children and adolescents enrolled in a health maintenance organization. Journal of Allergy and Clinical Immunology 2004; 113: 536-9.

\section{S Sri Ranganathan ${ }^{1}$, S A C Senadeera ${ }^{1}$, R Balasubramaniam ${ }^{1}$, R Fernandopulle ${ }^{1}$}

${ }^{1}$ Department of Pharmacology, Faculty of Medicine, University of Colombo, Sri Lanka.

Correspondence: SSR, e-mail: <sshalini14@hotmail.com>. Received 14 December 2010 and revised version accepted 15 March 2011. Competing interests: none declared. 